\title{
The association of diabetes and tuberculosis: impact on treatment and post-treatment outcomes
}

\author{
Roshani N Sanghani, ${ }^{1}$ Zarir F Udwadia ${ }^{2}$
}

\begin{abstract}
One woe doth tread upon another's heel, So fast they follow. William Shakespeare. Hamlet. Act 1V Scene V11
\end{abstract}

We are approaching a day and age where the terms communicable and noncommunicable diseases are taking on a new meaning. In the epidemiology and global public health space we track the top five causes of death to measure how well we are doing in combating illness. ${ }^{1}$ The idea that tuberculosis (TB) and diabetes mellitus (DM) share a synergistic relationship has been creating a growing stir around the world. This is so in the developed world where non-communicable diseases remain the leading cause of death and the developing world where the 'diabesity' epidemic is rapidly expanding to possibly overshadow the traditional killers from communicable disease. ${ }^{2}$ Of what relevance are these classifications in a patient unfortunate enough to have TB and DM? What, if anything, should we do differently in our clinics when a classical non-communicable illness like DM accelerates and amplifies the course of an age-old, communicable infection like TB? As the dreaded Mycobacterium grows deadlier with time, none of us want to see it develop new allies. The study published by Jimenez-Corona et $a l^{3}$ in this edition of Thorax suggests we have reason to be concerned. It also needs to be lauded upfront for being a major effort at identifying, exploring and characterising the TB-DM connection. They present a 15 year prospective follow-up of this growing area of concern. Indeed in the years since they started collecting data, numerous reports have emerged addressing the same concept in India, ${ }^{4}$ Brazil $^{5}$ and the US-Mexico border. $^{6}$ The study by Jiminez-Corona has several merits worth noting. They followed a large sample size totalling 1262 patients with pulmonary TB (PTB), and found a high prevalence of DM of 29.63\%.

\footnotetext{
'Department of Endocrinology, Hinduja Hospital and Research Center, Mumbai, India; ${ }^{2}$ Department of Pulmonary Medicine, Hinduja Hospital and Research Center, Mumbai, India
}

Correspondence to Dr Zarir F Udwadia, Department of Pulmonary Medicine, Hinduja Hospital and Research Center, Hinduja Hospital, Mumbai 400020, India; zarirfudwadia@gmail.com
The follow-up was long enough to allow observation of various predefined clinically relevant TB outcomes. They were thus able to demonstrate that dual disease (DM+PTB) was more likely to be associated with increased morbidity: pulmonary cavities, delayed sputum conversion $>60$ days, higher probability of treatment failure, and higher recurrence and relapse rates. While most relapses were reactivation with the same genotype, in almost a fifth (19\%) of all cases reinfection occurred with a different genotype, indicating genuine spread, possibly from attendance by diabetics at clinics with high local TB prevalence. This confirmed again that healthcare settings should be considered high risk environments for disease spread for our vulnerable patients. What this study failed to establish is higher mortality from TB in their DM-TB cohort. While these patients had higher rates of non-TB related overall mortality, the authors were unable to establish TB as the direct cause of death despite worse outcomes, postulating that the non-TB causes of death occurred too early to allow enough time for $\mathrm{TB}$ related sequelae to take effect. This is at variance with data from other studies. Higher odds of death were seen in the study by Wang $^{7}$ who noted TB related deaths were nearly three times as high in patients with DM as in patients without DM. A systematic review of the literature by Baker ${ }^{8}$ showed the relative risk of death from among 23 unadjusted studies during TB treatment was 1.89 (95\% CI 1.52 to 2.36 ) which increased to an effect estimate of 4.95 (95\% CI 2.69 to 9.10) among the four studies that adjusted for age and other potential confounding factors. Further research is needed to clarify whether TB-DM is causative for true increase in mortality. The intuitive question that comes to mind is the correlation of overall DM duration and level of glycaemic control on adverse TB outcomes. In practice we have long known that all DM is not equal; long standing uncontrolled DM is likely to correlate with worse outcomes on all parameters, and the converse is expected for one who has short duration of DM with optimal glycaemic control. How does duration of DM affect TB outcomes-is it a dose-dependent effect? Several studies have looked at this, and glycosylated haemoglobin tends to be a useful parameter to use. Leung et $a l^{9}$ demonstrated in a large cohort of 42116 elderly persons aged 65 years and older that HbA1c above $7 \%$ had a three times increased hazard of active PTB than those with A1c below 7\%. Future studies should be directed at teasing out the relationship between poor glycaemic control and worse TB outcomes especially focusing on a younger cohort in developing countries where TB-DM is on the rise; positive correlation would help guide and enforce clinicians to aggressively control DM in these high risk groups with the main goal of improving TB outcomes. Further study will be needed to answer these questions. Based on current standards of established medical care, we will never be able to conduct a randomised trial looking at better versus worse diabetic control and its effect on TB outcomes. Current DM management guidelines expect us to achieve adequate glycaemic control for all; but maybe the actively TB infected patient needs even more close attention due to the risk of added morbidity.

Thus there is now incontrovertible evidence that $\mathrm{TB}$ and $\mathrm{DM}$ are linked in a danse macabre. Diabetes triples the risk of developing TB and this chronic noncommunicable disease has now emerged as a powerful risk factor for one of mankind's most ancient infections. A strong case can and should be made for integrating $\mathrm{TB}$ and $\mathrm{DM}$ care. All confirmed TB patients should be systematically screened for DM and all diabetics should be actively screened for $\mathrm{TB}$ at least when they are symptomatic. Is screening for TB among diabetics likely to yield results? In a systematic review of literature Jeon ${ }^{10}$ demonstrated that screening of diabetics yielded active TB prevalence rates ranging from $1.7 \%$ in Sweden to $36 \%$ in Korea with TB being more common (prevalence ratios as high as 21) in those with insulin dependence compared with those with milder DM. ${ }^{11}$ Screening would have higher yield in a country like India where TB prevalence is estimated at 283/ 100000 . In this high-prevalence setting, screening 90-350 people with DM would yield one or more cases of TB. DM patients should be questioned about chronic cough (lasting $>2$ weeks) at the time they are diagnosed with DM and ideally at each regular follow-up. Those with positive TB symptoms should be examined as per national guidelines.

Conversely, what about screening for DM among TB patients? When all the studies that screened for DM prior to TB treatment were looked at, the prevalence of DM ranged from $8.6 \%$ in Turkey to $19.8 \%$ 
in Pakistan. In the setting of Mexico with among the highest DM baseline prevalence rates of $10 \%$, it would be bad practice not to screen all $\mathrm{TB}$ patients for $\mathrm{DM}$ since screening as few as 2-10 TB patients would yield at least one additional case of DM. Indeed the prevalence of $\mathrm{TB}$ in screened DM patients is commensurate with estimates for other populations in which active case finding is recommended such as HIV-infected individuals, gold miners in South Africa and prisoners in developing countries. Questions remain about the optimal timing of screening for DM. Active infection with TB may temporarily elevate blood glucose levels and the timing and choice of screening methods remain important unanswered questions.

Acknowledging the importance of bi-directional screening for TB and DM the WHO and the International Union Against Tuberculosis and Lung disease (IUATLD) released a landmark document in 2011 acknowledging this association and calling for increasing integration between DM and TB control efforts. ${ }^{12}$ Without integrated care this burden of DM threatens to sabotage attempts to meet the United Nations Millennium Development Goals of TB control. At the same time additional funds are of course needed. India for example, has the highest burden of $\mathrm{TB}$ in the world (accounting for 20\% of the global incidence) and houses around 58 million diabetics. Unfortunately in India, the optimal spending needed for each patient with DM exceeds by three times the amount being currently spent and these additional costs would overwhelm most TB control programmes. ${ }^{13}$ Consider the South East Asia (SEA) region: Sullivan showed that if all the
3-5 million estimated cases of TB in the SEA region in 2010 were actively screened for DM, an additional 66 500-1 225000 diabetics would be detected. ${ }^{14}$ The cost of treating these additional diabetics through the 6-month duration of Directly Observed Therapy Short Course (based on estimates from India of US\$149 direct cost per patient) would amount to an additional cost of US\$5-92 million. ${ }^{15}$ The long term funding of DM care and the management of its complications after the TB is treated is an even more vexatious issue. Many unanswered questions remain but well conducted prospective studies like the one by Jimenez-Corona are beginning to illuminate the path.

Contributors Both authors contributed equally. Competing interests None.

Provenance and peer review Commissioned; internally peer reviewed.

To cite Sanghani RN, Udwadia ZF. Thorax 2013;68:202-203.

Received 28 December 2012

Accepted 28 December 2012

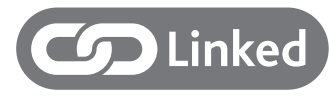

- http://dx.doi.org/10.1136/thoraxjnl-2012-201756

Thorax 2013;68:202-203.

doi:10.1136/thoraxjnl-2012-202976

\section{REFERENCES}

1 www.who.int/mediacentre/factsheets/fs310/en/index. html (accessed 14 Oct 2012).

2 Chen L, Magliano DJ, Zimmet PZ, et al. The worldwide epidemiology of type 2 . diabetes mellitus - present and future perspectives. Nat Rev Endocrinol 2011;8:228-36.
3 Jiminez-Corona ME, Cruz-Hervert LP, Garcia-Garcia L, et al. The association of diabetes and tuberculosis: impact on treatment and post-treatment outcomes. Thorax Published Online First: 18 December 2012. doi:10.1136/thoraxjnl-2012-201756

4 Stevensen, Forouhi NG, Roglic G, et al. Diabetes and tuberculosis: the impact of the diabetes epidemic on tuberculosis incidence. BMC Public Health 2007;7:234

5 Lindoso AA, Waldman EA, Komatsu NK, et al. Profile of tuberculosis patients progressing to death, city of São Paulo, Brazil, 2002. Rev Saude Publica 2008;42:805-12.

6 Restrepo BI, Fisher-Hoch SP, Crespo JG, et al. Type 2 diabetes and tuberculosis in a dynamic bi-national border population. Rev Saude Publica 2008;42:805-12.

7 Wang $\mathrm{CS}$, Yang $\mathrm{CJ}$, Chen $\mathrm{HC}$, et al. Impact of type 2 diabetes on manifestations and treatment outcome of pulmonary tuberculosis. Epidemiol Infect 2009;137:203-10.

8 Baker MA, Harries AD, Jeon $C Y$, et al. The impact of diabetes on tuberculosis treatment outcomes: A systematic review. BMC Med 2011;9:81. http://www. biomedcentral.com/1741-7015/9/81 (accessed 26 Dec 2012).

9 Leung CC, Lam TH, Chan WM, et al. Diabetic control and risk of tuberculosis: a cohort study. Am J Epidemiol 2008;167:1486-94.

10 Jeon $C Y$, Harries AD, Baker MA, et al. Bi-directional screening for tuberculosis and diabetes: a systematic review. Trop Med Int Health 2010;15:1300-14.

11 Oscarsson PN, Silver H. Incidence and coincidence of diabetes mellitus and pulmonary tuberculosis in a Swedish county. Acta Tuberculosea Scandinavica 1961;160:23-48.

12 World Health Organization and The International Union Against Tuberculosis and Lung Disease. Collaborative framework for care and control of tuberculosis and diabetes. Geneva: WHO, 2011.

13 Anjana RM, Ali MK, Pradeepa R, et al. The need for obtaining accurate nationwide estimates of diabetes prevalence in India. Rationale for a national study on diabetes. Indian J Med Res 2011;133:369-80.

14 Sullivan T, Amor YB. The co-management of tuberculosis and diabetes: challenges and opportunities in the developing world. PLoS Medicine 2012;9:e1001269.

15 Kapur A. Economic analysis of diabetes care. Indian J Med Res 2007;125:473-82. 